\title{
Analysis of Hymenoptera venom allergy in own material. Clinical evaluation of reactions following stings, in patients qualified for venom immunotherapy
}

\author{
Andrzej Chciałowski, Michał Abramowicz, Jerzy Kruszewski \\ Department of Infectious Diseases and Allergology, Military Institute of Medicine, Warsaw, Poland \\ Adv Dermatol Allergol 2019; XXXVI (3): 302-307 \\ DOI: https://doi.org/10.5114/ada.2018.75607
}

\begin{abstract}
Introduction: Hymenoptera venom allergy (HVA) in some patients occurs with general symptoms involving respiratory and cardiovascular system with anaphylactic shock with constitutes a significant threat to life.

Aim: Assessment of the prevalence of HVA in our own material.

Material and methods: There were 498 patients after a general reaction to wasp and/or bee venom. The survey included questions: the type of stinging insect, body parts stung by insects, profession, frequency of stings by wasps and bees depending on professional activity, places of stings, clinical symptoms using the scale according to Muller, and the treatment following the sting. Among 498 patients, there were 281 women and 217 men.

Results: Wasp stings were more frequent and affected 382 (77\%) persons, while bee stings affected 116 (23\%) persons. Limbs constituted the most common area of the body stung by both wasps and bees. The sting was more frequent in rural areas and during summer rest. A severe systemic reaction (class III and IV according to Muller) occurred more often in people stung by bees. The most common medications included intravenous glucocorticosteroids, calcium preparations and antihistamines. Intramuscular Adrenaline injection was used in $48 \%$ of patients, while only $15 \%$ received its prescription as an emergency medication. Bee stings were the most common cause of severe systemic symptoms.

Conclusions: The treatment after the sting in a significant percentage of episodes still deviates from the recommendations of the guidelines, especially in the field of adrenaline recommendations for patients in case of a resting by an insect
\end{abstract}

Key words: Hymenoptera venom allergy, immunotherapy, anaphylaxis.

\section{Introduction}

Allergy to the hymenoptera venom (Hymenoptera venom allergy - HVA) is a separate branch of modern allergology, which is becoming more and more popular not only among medical practitioners and allergologists. It usually occurs along with general symptoms involving respiratory and cardiovascular systems with anaphylactic shock, which constitutes a significant threat to life.

The incidence of HVA constituting a life-threatening risk is estimated at $0.9-3.4 \%$ in children and $5.0-8.9 \%$ in adults. It depends on individual predispositions from the geographical region, climate, season and profession [1, 2]. In the ECAP study carried out in Poland, $2-3 \%$ of respondents declared hypersensitivity to insect venom [3]. In the study by Nittner-Marszalska as many as $8.9 \%$ of the inhabitants of Lower Silesia declared a systematic anaphylactic reaction after a sting [4]. Deaths are observed sporadically. Literature data report their occurrence with the frequency of $0.3-0.48$ cases per 1 million inhabitants/ year, which constitutes approx. $20 \%$ of deaths in the course of anaphylaxis for other reasons [5-7]. The most dangerous part of the body to be stung is the region of the tongue, throat, mouth or neck [8].

Degrees of severity of the anaphylactic reactions are given on various scales, e.g. Muller, Sipson or Ring and Messman [7, 9]. Treatment of anaphylactic shock first of all involves applying adrenaline in the form of intramuscular injection [10, 11]. Retrospective studies of Oropeza et al. demonstrated that adrenaline was administered

Address for correspondence: Michał Abramowicz PhD, Department of Infectious Diseases and Allergology, Military Institute of Medicine, 128 Szaserów St, 04-141 Warsaw, Poland, phone: +48 2281029 09, e-mail: mabramowicz@wim.mil.pl Received: 25.03.2018, accepted: 30.03.2018. 
in only $54 \%$ of cases with moderate and severe anaphylaxis, whereas in the German study by Bayer et al. it was only $29.6 \%$ [12]. It should be mentioned that antihistamine drugs and glucocorticoids were applied in almost every case [13]. A systemic reaction after the sting is an indication to extend the diagnosis in terms of qualifications for VIT.

\section{Aim}

The aim of the present study was to perform an indepth retrospective analysis of serious episodes after insect stings in patients referred to the Allergy Clinic of the Department of Infectious Diseases and Allergology of the Military Institute of Medicine in Warsaw in the years 2003-2017 to qualify for immunotherapy against insect venom (venom immunotherapy - VIT).

\section{Material and methods}

Out of approximately 58 thousand patients of the Clinic of Allergology at the Clinic of Infectious Diseases and Allergology of the Military Medical Institute in Warsaw in the years 2003-2017, the retrospective analysis covered medical documentation of patients referred with a preliminary diagnosis of HVA. Out of the 1740 (3\%) patients suspected of an insect venom allergy, 498 (0.85\%) patients with a documented history of anaphylactic reaction to a wasp and/or bee venom confirmed by positive skin tests were qualified for further analysis.

This group's data were analyzed to determine the following:

- kind of stinging insect (wasp, bee),

- part of the body stung by the insect (head, trunk, limbs),

- profession (professions at risk of insect sting: beekeeper, farmer, forester, fireman, etc.),

- impact of the patient's activity (working, resting) on the kind of stinging insect (wasp and bee),

- place where the stinging occurred (city, village),

- clinical symptoms that constitute the framework to qualify using the severity scale according to Muller Table 1,
- treatment after sting (adrenaline, systemic glucocorticosteroids, antihistamines, calcium preparations).

\section{Ethics}

The study was approved by the Military Institute of Medicine Ethics Committee.

\section{Statistical analysis}

Baseline characteristics are presented as numbers and percentages. The arithmetic mean and standard deviations (SD) were calculated for two patient groups as the central measure. To compare groups of patients we used the analysis of variance tests, or $\chi^{2}$ tests. Odds ratios were calculated to assess the likelihood of a body part being stung, occupation from the list of risky professions, place of sting and rate of sting depending on occupational activity. For all the tests $p$-values of $<0.05$ were considered statistically significant.

\section{Results}

Among 498 analyzed patients aged $43.17 \pm 14.76$, there were 281 (56.4\%) women aged $44.69 \pm 14.61$ and 217 (43.6\%) men aged $41.20 \pm 14.76$.

Wasp stings were reported by 382 (77\%) patients at the age of $43.31 \pm 14.69$ years, which was significantly $(p<0.05)$ more frequent than bee stings, reported by 116 (23\%) people at the age of $42.70 \pm 15.04$ years.

Limbs were the most often stung part of the body, by both wasps and bees, followed by the head and torso. Statistical analysis did not show any significant preference for the part of the body that was stung by wasps and bees respectively, as illustrated in Figure 1.

Bees stung significantly $(p<0.05)$ more often people performing a profession at risk of insect stinging compared to wasps. Out of 246 (100\%) of the surveyed patients, in 70 (29\%) the episode causing anaphylaxis after the sting took place in urban areas ( $90 \%$ stung by a wasp and $10 \%$ by a bee), and 176 (71\%) in rural areas (including $68 \%$ of people stung by a wasp and $32 \%$ by a bee). The results are shown in Figure 2.

Logistic regression analysis showed 4 -fold $(95 \% \mathrm{Cl}$ : 1.75-9.54) higher risk of wasp sting in the city.

Table 1. Grading of anaphylaxis according to severity of clinical symptoms (Mueller 1966)

\begin{tabular}{|c|c|}
\hline Grade & Signs and symptom \\
\hline 1. Slight general reaction & Generalized urticaria, itching, malaise and anxiety \\
\hline 2. General reaction & $\begin{array}{l}\text { Any of the above plus two or more of the following: generalized edema; constriction in chest; } \\
\text { wheezing; abdominal pain; nausea and vomiting; and dizziness }\end{array}$ \\
\hline 3. Severe general reaction & $\begin{array}{l}\text { Any of the above plus two or more of the following: dyspnea; dysphagia; hoarseness or thickened } \\
\text { speech; confusion; and feeling of impending disaster }\end{array}$ \\
\hline 4. Shock reaction & $\begin{array}{l}\text { Any of the above plus two or more of the following: cyanosis; fall in blood pressure; collapse } \\
\text { incontinence; and unconsciousness }\end{array}$ \\
\hline
\end{tabular}


Patient activity was significantly $(p<0.0004)$ associated with the frequency of stings. Most of them were accidental and concerned 194 (78\%) people who were enjoying summer rest ( $80 \%$ by wasps and $20 \%$ by bees). The remaining 53 (22\%) people were stung during work ( $57 \%$ by a wasp and $43 \%$ by a bee). The fact of performing work increased the risk of being stung by a bee three-fold (95\% Cl: 1.59-5.83). The results are illustrated in Figure 3.

Severe generalized reaction with systemic symptoms affecting respiratory and cardiovascular systems (class III and IV according to Muller) occurred in 260 patients stung by wasps (68\%) out of $382(100 \%)$ stung by this insect, and 81 (70\%) patients out of $116(100 \%)$ stung by bees, as shown in Figure 4.

As mentioned earlier, limbs were the most common part of the body stung by both the insects. However, there was a significant ( $p=0.0001$ ) relationship between the head sting and the fourth class of severity according to Muller, regardless of the type of stinging insect.

In patients with documented anaphylactic reactions after stings by wasps and bees, the most frequent (65\%

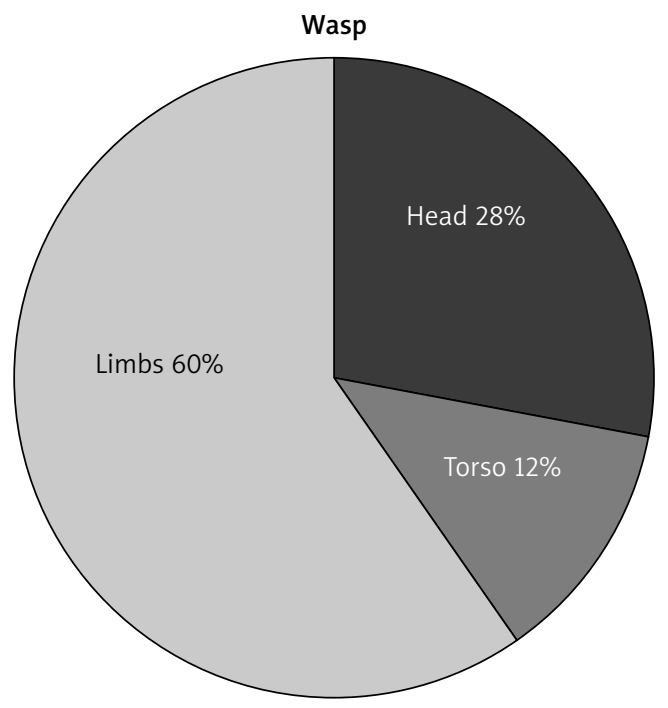

Figure 1. Body part and the percentage of stings

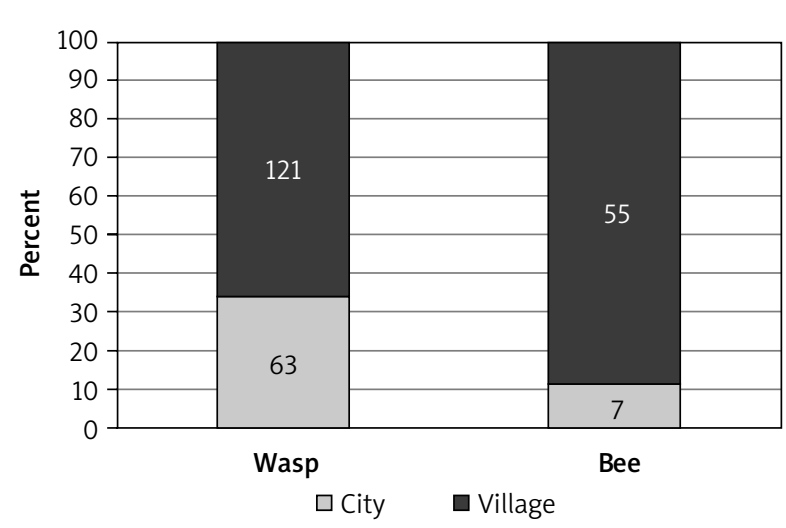

Figure 2. A place of insect sting occurrence of episodes) drug used by medical staff of Primary Health Care or Emergency Department facilities were systemic glucocorticosteroids. Adrenaline in intramuscular injection was used only in $48 \%$ of patients, most often in the fourth degree of severity according to Muller. Only in 15\% of patients was adrenaline prescribed after the episode as a rescue medication in the event of a re-sting. Calcium preparations were used intravenously in $22 \%$ of patients, while almost all received antihistamine in the form of intravenous, intramuscular or oral injection. The results are shown in Figure 5.

\section{Discussion}

Stings caused by Hymenoptera belonging to the bee family Apidae, including honey bees and bumblebees, and Vespidae - wasps and hornets - are quite common in areas with a warm, temperate climate. According to Bilo et al. [2, 7] about $56.6-94.5 \%$ of people have been stung by these insects at least once in their lifetime. In most cases, transient and mild local

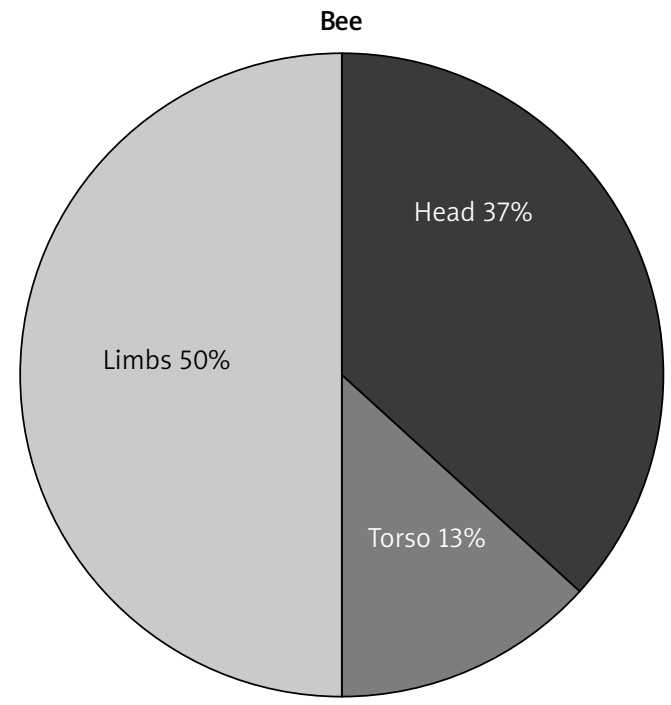

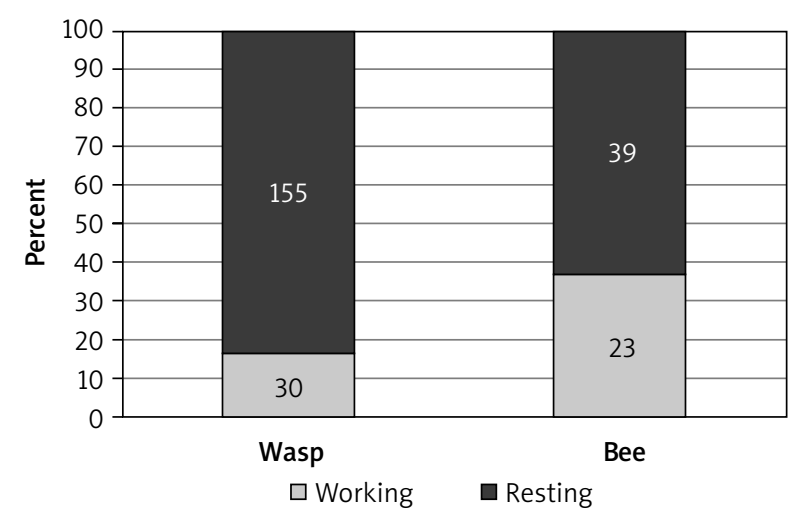

Figure 3. The frequency of stings depending on the patient's activity 

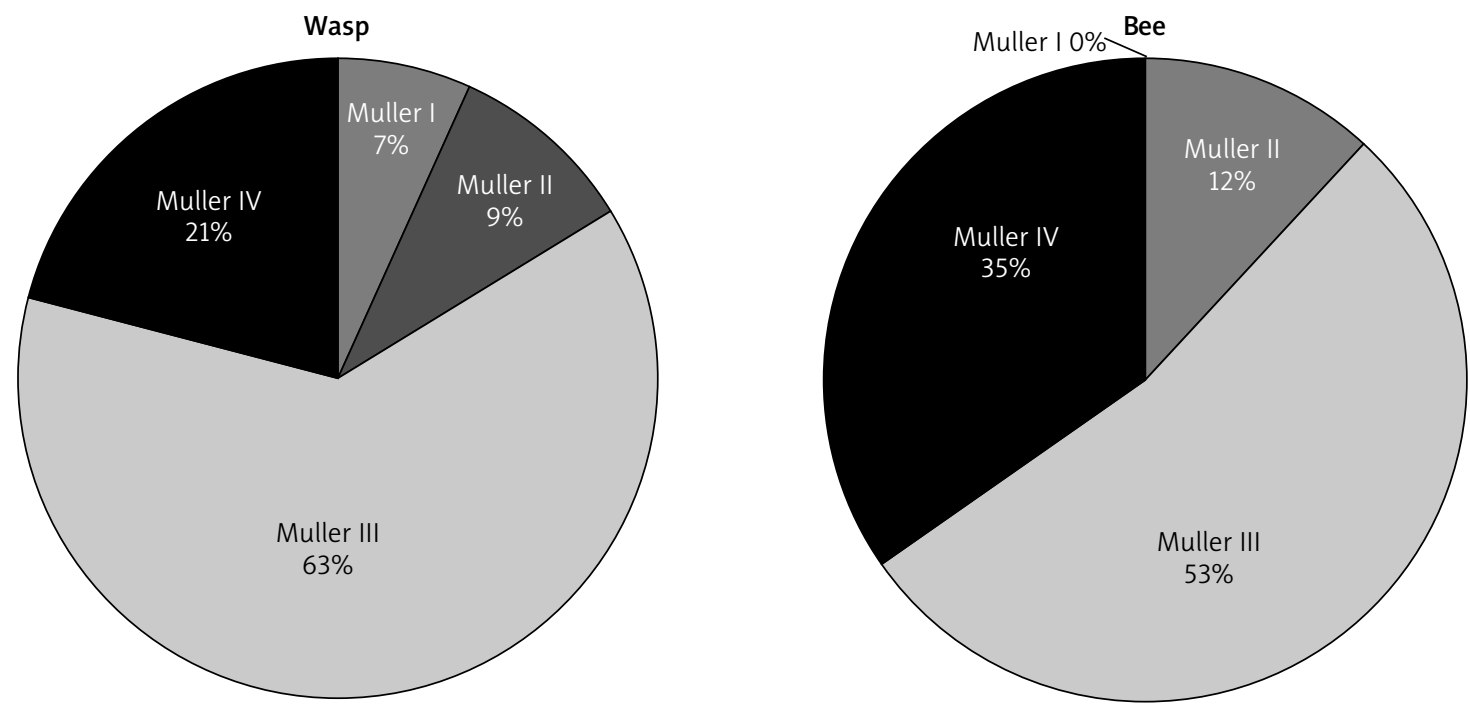

Figure 4. Percentage of people with clinical symptoms according to Muller scale

reactions occur, and they disappear spontaneously or require short-term local treatment. In some people, they lead to a large local reaction (LLR) in 5-26\% of cases or a generalized (severe allergic reaction - SAR) anaphylactic reaction that is a direct threat to life in the mechanism of shock. In Europe, anaphylaxis affects from $0.3 \%$ to $7.5 \%$ of stung individuals [11], and according to national data from the ECAP survey and conducted by Nittner-Marszalska in Lower Silesia SAR was reported by $2-3 \%$ and $8.9 \%$ of respondents respectively $[4,14]$. It seems that one of the important factors leading to their occurrence may be the type of insect, sex or the body region subjected to a sting [15]. Other factors also remain significant. They include the following: allergic diseases (bronchial asthma, atopic dermatitis), co-morbidities, especially cardiovascular and respiratory diseases, mastocytosis and/or elevated serum tryptase concentration, as well as the administered drugs: angiotensin-converting enzyme inhibitors (ACE inhibitor) and $\beta$-blockers [2].

Available publications consider age as one of the elements resulting in an increased risk of severe reactions $[2,5]$. In the studies conducted by Artz et al., serious allergic reactions were observed in 189 (71.9\%) people over 40 years of age compared with 74 (28.1\%) younger persons [6-8]. Although stinging by wasps was more frequent compared to bees, there was no significant relationship between the type of stinging insect and the severity of the symptoms. The studies by Kalyoncu et al. and Stoevesandt et al. did not reveal any significant differences in the occurrence of severe general reactions depending on gender [4]. Similarly, in the current analysis, although women were more often stung, no significant differences were observed between sex and the type of the stinging insect. However, more severe reactions

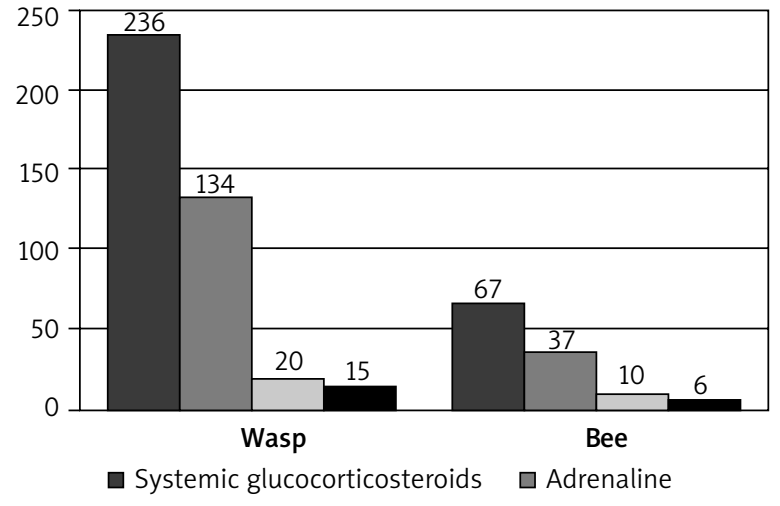

$\square$ Calcium preparations Adrenaline autoinjector prescription

Figure 5. Type and frequency of treatment following a sting

on the Muller scale were demonstrated in people over 40 years of age.

It was found that bees stung people in the risk group significantly more frequently than wasps, but this was not a significant reason for the occurrence of severe reactions requiring proper therapeutic treatment. This is fully understandable, considering the professions that predispose to an increased frequency of stinging (especially beekeepers and farmers). Münstedt et al. analyzing a population of German beekeepers found a negative relationship between the severity of symptoms and age, body mass index (BMI) and the number of hives in the apiary, and no gender relationship was observed. A significant negative correlation was also found between 'working years' and the likelihood of an allergic reaction [16].

The present study demonstrates that most of the stings occurred in rural areas and during summer rest and they were more often caused by wasps. This is understandable as spring and summer seasons favoring ac- 
tive rest are periods of increased insect flights. Moreover, sunny and warm weather favors wearing of light clothing leaving most parts of the body exposed, and the use of cosmetics and the consumption of sweet drinks and meals in the open attract insects. Clarc and Camargo in their proposal of educational recommendations for patients take into account the necessity of using appropriate clothing during outdoor activities, avoiding fruit plantations and meadows (grass), and taking precautions in the use of aromatic cosmetics and soaps and when consuming sweet meals [17].

It is thought that stings in the head, neck or mouth area result in more severe reactions than stings in other parts of the body $[7,8]$. This may be due to the presence of well-vascularized soft tissues in the area that facilitate the penetration of venom into blood vessels, the binding of specific IgE to the FceRI receptors of the cell wall of plasma cells and basophils with the rapid mobilization of acute allergic mediators. Moreover, developing swelling can lead to airway obstruction. Our own material demonstrates that stings in the head were more frequent among men than women, which can be fully explained by the presence of long, dense hair in women compared to men. This is consistent with the report by Artz et al. where head stings were significantly more common in men than in women (62\% vs. 38\%) [8]. In the analyzed group, there was a significant correlation between head stings and systemic reactions (Muller IV) and the need for appropriate treatment. This somewhat contradicts the reports of those authors who have shown that head stings were the cause of a severe reaction in only $13.3 \%$ of cases. In $87 \%$ of cases they occurred after stinging in the torso, followed by $39.1 \%$ in upper limbs and 30\% in lower limbs [6].

Treatment of an anaphylactic reaction must be implemented immediately after its diagnosis as it is an immediate threat to life $[6,18,19]$. Guidelines for the treatment of severe allergic reactions and anaphylactic shock clearly recommend the immediate administration of epinephrine in an intramuscular injection together with an intravenous infusion of fluids [20]. In the analyzed material, a severe allergic reaction was observed in 260 people after a wasp sting and in 81 people stung by bees. Both in patients after a wasp sting as well as those after a bee sting with a documented anaphylactic reaction, the most common drugs besides intravenous fluid infusions and oxygen therapy in $65 \%$ of cases involved glucocorticosteroids in intravenous injection. Adrenaline in the form of an intramuscular injection was used only in $48 \%$ of patients.

Our results agree with the reports of other researchers. As demonstrated by Oropeza et al. in a Danish 5-year retrospective analysis of 273 cases of a clinical response after insect stings, adrenaline was used in $54 \%$ of moderate and severe reactions according to the Sampson scale, while intravenous glucocorticosteroids were used in $88 \%$ and antihistamines in $91 \%$ of cases, by staff of the outpatient teams (Ambulance) and Emergency Department [12].

Previous Scandinavian research demonstrated that adrenaline in severe anaphylactic reactions was used in $78-80 \%$ of cases by the anesthetic team of the Anesthesiologist Staff of the Ambulance Helicopter [21]. Brown et al. in 2001 presented the results of an analysis of the treatment of severe anaphylactic reaction after ingested drugs, food and insect stings, at Australian Emergency Departments (EDs). Of 142 patients only $18 \%$ received adrenaline before reaching the ED and $27 \%$ during their stay at the ED. Glucocorticoids, on the other hand, were administered to $16 \%$ and $75 \%$ respectively. Insect stings caused $18 \%$ of cases of anaphylactic reaction. In total, adrenaline was administered to only $45 \%$ of patients, glucocorticosteroids to $78 \%$ and antihistamines to $85 \%$ of patients [19].

In a Swiss study covering a 3-year observation period, Helbling et al. demonstrated that out of 215 patients suffering from an anaphylactic shock, insects were the cause of its occurrence in 133 (59\%) cases and in 210 (98\%) ED intervention was necessary, and only half of them received adrenaline [18]. In another analysis conducted by Clarc and Camargo, among 46 people with a severe reaction at the $E D$, only $18 \%$ received adrenaline. Stings and the development of severe life-threatening reactions occur less frequently in children [17]. Tiyyagura et al., based on a retrospective study conducted in the years 2008-2010 among 202 children, found 218 cases of anaphylaxis in the course of food, inhalation and drug allergy, and only in 1\% following insect stings [22]. Only $36 \%$ received epinephrine at Emergency Medical Services (EMS), while some received it at schools from nurses or at doctors' offices.

Experiencing a severe anaphylactic reaction requires subsequently undergoing appropriate tests and, if necessary, qualification for specific immunotherapy. In anticipation of its implementation, it is important to protect patients against future reactions and to provide the socalled rescue set, consisting of adrenaline in the form of an automatic syringe (autoinjector) and antihistamine drugs [15, 20, 23]. The results of our analysis demonstrated that adrenaline was prescribed for home use as a rescue medicine in only $15 \%$ of patients. Almost all patients in the analyzed study and in the cited studies received antihistamine in the form of intravenous, intramuscular injection or orally. Literature data show that this is a global problem. The authors, including Clark, report that after a severe anaphylactic reaction, only $27 \%$ of patients were prescribed adrenaline, and only $15 \%$ of them were explained the causes and course of the anaphylactic reaction. This may partly be due to the ignorance of some doctors regarding the possible dual-phase nature of the allergic reaction with the occurrence of the late phase a few hours after the sting. 
The analysis carried out in the present study also has several limitations. Its retrospective character and data obtained only from patients without or with scant medical records may have been the cause of incorrect classification of certain cases to individual degrees of severity of the course of the anaphylactic reaction. The available documentation lacked any information on the collection of material or determination of the number of mast cells in blood cells or serum tryptase concentration - therefore, it is not possible to determine in how many of them the reaction could have been increased by the presence of e.g. mastocytosis. All the patients, however, had skin tests performed and slgE concentrations determined, and some of them were qualified for specific allergen immunotherapy, which will be the subject of another report.

\section{Conflict of interest}

The authors declare no conflict of interest.

\section{References}

1. Bonifazi F, Jutel M, Biló BM, et al. Prevention and treatment of hymenoptera venom allergy: guidelines for clinical practice. Allergy 2005; 60: 1459-70.

2. Bilo MB, Bonifazi F. Epidemiology of insect-venom anaphylaxis. Curr Opin Allergy Clin Immunol 2008; 8: 330-7.

3. ECAP. Epidemiologia Chorób Alergicznych w Polsce (www. ecap.pl).

4. Nittner-Marszalska M, Liebhart J, Liebhart E, et al. Prevalence of Hymenoptera venom allergy and its immunological markers current in adults in Poland. Med Sci Monit 2004; 10: 324-9.

5. Nittner-Marszalska M, Cichocka-Jarosz E. Insect sting allergy in adults: key messages for clinicians. Pol Arch Med Wewn 2015; 125: 929-37.

6. Bilo MB. Anaphylaxis caused by hymenoptera stings: from epidemiology to treatment. Allergy 2011; 66 Suppl 95: 35-7.

7. Bilo MB, Bonifazi F. The natural history and epidemiology of insect venom allergy: clinical implications. Clin Exp Allergy 2009; 39: 1467-76.

8. Artz L, Bucanovic D, Schwarz I, et al. Hymenoptera stings in the head region induce impressive but not severe sting reaction. Allergy 2016; 71: 1632-4.

9. Ciszowski K, Miętka-Ciszowska A. Hymenoptera stings. Przegl Lek 2007; 64: 282-9.

10. Muller U, Mosbech H, Blaauw P, et al. Emergency treatment of allergic reactions to Hymenoptera stings. Clin Exp Allergy 1991; 21: 281-8.

11. Golden DB, Moffitt J, Nicklas R, et al.: Joint Task Force on Practice Parameters; American Academy of Allergy, Asthma \& Immunology (AAAAI); American College of Allergy, Asthma \& Immunology (ACAAI); Joint Council of Allergy, Asthma and Immunology. Stinging insect hypersensitivity: a practice parameter update 2011. J Allergy Clin Immunol 2011; 127: 852-4.

12. Oropeza AR, Mikkelsen S, Bindslev-Jensen C, et al. Pre-hospital treatment of bee and wasp induced anaphylactic reactions: retrospective study. Scand I Trauma Resusc Emerg Med 2017; 25: 4.

13. Beyer K, Eckermann O, Hompes S, et al. Anaphylaxis in an emergency setting - elicitors, therapy and incidence of severe allergic reactions. Allergy 2012; 67: 1451-6.
14. Nittner-Marszalska M, Liebhart J, Dor-Wojnarowska A. Sexrelated clinical aspects in insect venom anaphylaxis. Int J Immunopathol Pharmacol 2015; 28: 187-93.

15. Bilo MB. Diagnosis of Hymenoptera venom allergy. Allergy 2005; 60: 1339-49.

16. Münstedt K, Hellner M, Winter D, et al. Allergy to bee venom in beekeepers in Germany. J Investig Allergol Clin Immunol 2008; 18: 100-5.

17. Clarc S, Camargo CA. Emergency treatment and prevention of insect-sting anaphylaxis. Curr Opin Allergy Clin Immunol 2006; 6: 279-83.

18. Helbling A, Hurni T, Mueller UR, Richler WJ. Incidence of anaphylaxis with circulatory symptoms: a study over a 3-year period comprising 94000 inhibitans of the Swiss canton Bern. Clin Exp Alllergy 2004; 34: 285-90.

19. Brown A, McKinnon D, Chu K. Emergency department anaphylaxis; a revive of 142 patients in a single year. J Allergy Clin Immunol 2001; 108: 861-6.

20. Kemp SF, Lockey RFF, Simons ER; the World Allergy Organization ad hoc Committee on Epinephrine in Anaphylaxis. Epinephrine: the drug of choice for anaphylaxis. A statement of the World Allergy Organization. Allergy 2008: 63: 1061-70.

21. Soreide E, BuxrudT, Harboe S. Severe anaphylactic reactions outside hospital etiology, symptoms and treatment. Acta Anesthesiol Scand 1988; 32: 339-42.

22. Tiyyagura GK, Arnold L, Cone DC, et al. Pediatric anaphylaxis management in the prehospital setting. Prehosp Emerg Care 2014; 18: 45-50.

23. Przybilla B, Ruëff F. Insect stings. Clinical features and management. Dtsch Arztebl Int 2012; 109: 238-48. 